\title{
MODELOS CIENTÍFICOS, TEORÍA SOCIOLÓGICA Y EL PROBLEMA MACRO-MICRO
}

Renate Mayntz ${ }^{1}$

\begin{abstract}
RESUMEN
Las relaciones metodológicas entre las ciencias naturales y las sociales son objeto de un renovado debate en el seno de estas últimas. En las dos décadas pasadas, la evolución en las primeras ha producido signos suficientes para hablar de la emergencia de un nuevo paradigma que diferiría del patrón fisicalista newtoniano. Desde la filosofía, la teoría y la metodología sociológica se ha abogado para que esos avances de las ciencias naturales tuvieran su repercusión en las sociales. Renate Mayntz dirigió una investigación en el Instituto Max Planck cuyos principales resultados se reflejan en este artículo. Su ponderación pesimista acerca de las posibilidades de trasvase metodológico de un ámbito a otro no impide que valore adecuadamente aquellos ámbitos de investigación sociológica donde sí se ha dado una influencia metodológica efectiva.
\end{abstract}

Por más que las disciplinas científicas pretendan configurarse como unidades grupales limpiamente delimitadas, difícilmente logran formar sistemas cerrados desde un punto de vista cognitivo. Al contrario, la historia evolutiva de la ciencia está plagada de casos de cruces transfronterizos y de enriquecimiento recíproco. Dentro de estos cruces pueden distinguirse varios tipos de relaciones científicas interdisciplinares: joint ventures constituidas por varias disciplinas que trabajan juntas en un mismo tema; intentos reduccionistas o, a

1 (N. del T.) El original se encuentra en W. Zapf (ed.), Die Modernisierung moderner Gesellschaften, Frankfurt/Main, Campus Verlag, 1991. 
la inversa, imperialistas de rehacer las fronteras, $y$, finalmente, la utilización en una disciplina de métodos, conceptos y modelos teóricos tomados de otra. En este trabajo trataremos únicamente de este último tipo de relación interdisciplinar $^{2}$; y, más concretamente, sólo nos ocuparemos de la utilización por parte de las ciencias sociales de modelos tomados de las ciencias naturales. El caso contrario no nos interesa, y no porque no haya habido experiencias históricas de ello, empezando por Darwin, por ejemplo, sobre el que influyó el filósofo y sociólogo Spencer, o ya en nuestros días la influencia que la teoría de juegos ejerce sobre la actual biología evolutiva.

La relación de la sociología con las ciencias naturales ha sido siempre ambivalente. Ha habido muchos que, siguiendo a Dilthey, han visto la ciencia natural como «una tarea baja y mezquina», que sólo "atiende a la repetición vacía y yerma del mecánico ciclo natural» (citamos por Queisser, 1987: 13). Sin embargo, al mismo tiempo, las ciencias naturales han servido desde un principio como modelo no sólo en el plano metodológico, sino también en el teórico. En este sentido, los intentos de fundar una Mecánica o una Física Social, vistos en conjunto, jugaron un papel más pequeño que los que tomaron como modelo a la biología. Esto vale no sólo para Spencer y los desarrollos teóricos que se basan en él, sino también para Durkheim, quien, aunque insistió en ver los hechos sociales como cosas, a nivel semántico era más bien deudor de supuestos organicistas.

Últimamente tiene uno la impresión de que los sociólogos, tras una fase de autorreferencialidad, se están asomando con renovado interés sobre las lindes que delimitaban la disciplina para otear al campo de las ciencias naturales. A veces, incluso, puede llegar al extremo de que todo sociólogo que quiera pasar por epistemológicamente vanguardista tiene que manejar con soltura términos como sinergética, autopoiesis y caos determinista, así como mostrarse familiarizado con las obras de Prigogine, Haken, Maturana, Thom y Eigen. Obviamente, esto no afecta por igual a todos los ámbitos de nuestra disciplina. Los menos afectados son las sociologías empíricamente especializadas ${ }^{3}$; de ellas, sólo la sociología de las organizaciones, en la que últimamente juegan un papel destacable conceptos procedentes de la biología evolutiva, constituye una cierta excepción. Por el contrario, la influencia de los modelos científico-naturales se nota especialmente en el ámbito de la teoría macrosociológica, particularmente en el intento de modelización formal y matemática.

Los métodos, conceptos y teorías físico-matemáticas que atraen la atención de los sociólogos que trabajan en esos dos ámbitos son a primera vista muy heterogéneos. Las relaciones entre el depredador y la presa y el hiperciclo de Eigen se mezclan con la teoría de catástrofes y la teoría del caos, ambas matemáticas; a ellas se suma la teoría de las estructuras disipativas de Prigogine y la de la autoorganización de Von Foerster; la teoría sinergética de Haken y la teo-

2 Considerar que este tipo es posible presupone una concepción de la sociología en cuanto ciencia que no comparten los representantes de un enfoque hermenéutico radical.

3 (N. del T.) Traducimos así la expresión que la autora utiliza, Bindestrich-Soziologien; literalmente, «sociologías de algo (cine, tercera edad, etc.)». 
ría autopoiética de Maturana. Visto con detenimiento, todos estos planteamientos están en armonía y pueden ser considerados como componentes de una macroteoría hipotética de sistemas dinámicos. El nuevo interés de los sociólogos en modelos científico-naturales depende de eso que hoy nos gusta apostrofar como cambio en el pensamiento científico-natural: el abandono de la imagen del mundo de la mecánica newtoniana y el interés creciente por investigar procesos no lineales en sistemas inestables, que ya no son vistos como molestas excepciones, sino cada vez más como la regla del acontecer. Por esta inclinación al estudio de la dinámica de los sistemas no lineales por parte de las ciencias naturales en sentido estricto, esto es, especialmente la física y la química, es por lo que han ganado interés para la sociología y pueden competir hoy con el atractivo de las ciencias de la vida. Que haya un renacimiento de conceptos científico-naturales en la teoría sociológica, y en la medida en que esto se esté dando de hecho, es un indicador, parcial al menos, de la relevancia creciente que el pensamiento científico-natural contemporáneo tiene para la sociología. Pero esto no sería más que una afirmación vacía de contenido si no pudiésemos precisar con mayor exactitud para qué pueden ser de utilidad los modelos dinámicos de sistemas no lineales para el conocimiento sociológico. Sólo entonces podremos estimar la importancia de los conceptos científiconaturales recientes para el desarrollo de una teoría sociológica con capacidad explicativa.

Para poder contestar a esta cuestión con conocimiento de causa, serían necesarios suficientes conocimientos acerca de las teorías científicas mencionadas anteriormente. Por razones de tiempo, no me queda sino presuponer esos conocimientos ${ }^{4}$, aunque por lo menos quisiera referirme a algunos puntos especialmente importantes para las reflexiones que siguen.

Bajo la etiqueta de dinámica de sistemas no lineales se incluyen tanto teorías de carácter analítico-formal como sustantivas. En el grupo de las primeras hay que destacar la teoría matemática de catástrofes de René Thom y el análisis del caos determinista. En ambas, las discontinuidades o saltos de una fase a otra juegan un papel central, especialmente el salto repentino de orden a caos. Características importantes de estos procesos son la existencia de valores umbral, la dependencia de los acontecimientos respecto de una trayectoria y la posibilidad de bifurcaciones, es decir, de posibles procesos alternativos bajo determinadas circunstancias. Las teorías sustantivas se ocupan principalmente de procesos de emergencia espontánea de orden, de la fase de transición que sigue a una ruptura del orden y que conduce a una nueva situación de desequilibrio. Los fenómenos a los que se refieren estas teorías son extraordinariamente variados. Van desde el ferromagnetismo, la superconductividad y el láser a la formación de colonias de células móviles y a los procesos autocatalíticos responsables de la génesis de las moléculas proteicas autorreproductoras, pasando

Una exposición detallada puede verse en Renate Mayntz (1990). Véanse ahí también referencias bibliográficas más detalladas. 
por procesos hidrodinámicos, relojes químicos y las manchas en la piel del leopardo. No obstante, todos estos procesos, vistos desde un conveniente nivel de abstracción, pueden ser reunidos en un paradigma común. Tanto si, como parece ser la inclinación de los sociólogos, nos referimos a él con el término de autoorganización siguiendo a Von Foerster, como de estructuras disipativas siguiendo a Prigogine o de sinergética siguiendo a Haken, los elementos esenciales de este paradigma común pueden ser resumidos brevemente de este modo:

1. Los procesos autoorganizativos tienen lugar propiamente en sistemas constituidos por una gran cantidad de elementos.

2. Tales sistemas se encuentran en estado de inestabilidad termodinámica.

3. Los sistemas toman de su entorno la energía que utilizan en el proceso de autoorganización.

4. El orden o la estructura que se forma no se produce por causas externas, sino por la interacción entre los elementos del sistema. Este orden emergente representa una macrocualidad nueva del sistema.

5. Tales procesos autoorganizativos son, intrínsecamente, de naturaleza no lineal.

La teoría autopoiética de Varela y Maturana puede ser vista como otra variante de los procesos autoorganizativos. Mientras que en los sistemas físicoquímicos se trata exclusivamente de la formación de la estructura endógena, la autopoiesis implica la autoproducción sistémica. Dicho de otro modo, el sistema produce sus propios elementos, lo cual constituye una característica de los sistemas vivos, de la vida orgánica. Este paradigma autoorganizacional ampliado a procesos de autoproducción puede ser considerado, finalmente, como parte de un modelo evolutivo más general, presuponiendo que nos alejemos de la concepción tradicional de que la evolución es un proceso determinado sólo por selectores externos.

No es éste el lugar para una descripción detallada de las distintas influencias en las ciencias sociales a que han dado lugar estos modelos científicos ${ }^{5}$. Resumiendo, a grandes rasgos podemos encontrar dos tipos de influencias, que podríamos caracterizar como formal y discursiva. Cada una es representativa de uno de los dos ámbitos de recepción señalados antes. El primer tipo de influencia se da en el ámbito de la modelización formal, especialmente matemáti$c a$, de procesos sociales, donde no se trata tanto de teorías científicas referidas a algún objeto natural, sino más bien de procedimientos y modelos matemáticos. Lo interesante aquí es la estructura formal de estos procesos y la posibilidad de formularla matemáticamente. Según esto, se toman como referencia determinados desarrollos formales de dinámicas no lineales, como la teoría

5 Para ello puede verse también Mayntz (1990). 
matemática de catástrofes de R. Thom (Thom, 1972) o el análisis científico de procesos físicos de autoorganización que incluso llegan a ser formulados matemáticamente de modo parcial. Esto último vale especialmente para la sinergética de Haken (Haken, 1978). Los conceptos generales de transición de fase, de ruptura estructural o de orden espontáneo en sistemas inestables pueden haber actuado como estímulo, pero no son decisivos para ese tipo de influencia que se centra en aspectos formales de métodos y modelos.

En el ámbito de la teoría macrosociológica se da justamente lo contrario, pues ahí interesa tanto el análisis formal de sistemas dinámicos no lineales como el análisis de los procesos mismos que son objeto de teorías científicas como la evolución, la autopoiesis o la autoorganización. En el caso de la recepción discursiva de teorías científicas referidas a un objeto, no puede hablarse prácticamente de una recepción en sentido estricto. No obstante, hay intentos de buscar correspondencias sociológicas con una literalidad vergonzosa respecto de conceptos clave de las teorías científicas tomadas como referencia sin prestar la suficiente atención al problema del isomorfismo. Dentro de la recepción puramente metafórica se trata, en el mejor de los casos, de innovaciones semánticas que no añaden nada a lo que ya sabemos de la realidad social, pues lo que se hace es reescribir hechos conocidos en una nueva terminología. En el caso de la recepción discursiva más seria y teóricamente más ambiciosa que se ha dado esencialmente en el ámbito de la teoría de sistemas y enfoques teóricos afines, no se intenta aplicar sin más una teoría científica ya desarrollada a fenómenos sociales. Luhmann (1984), por ejemplo, parte de un modelo teórico sistémico con un alto grado de generalización, con lo que para él ni siquiera se plantea la necesidad de discutir la posibilidad y los límites de una transferencia teórica desde el ámbito científico natural. Como ilustra este ejemplo, la recepción de modelos científicos en el ámbito de la teoría sociológica se produce de modo característico por generalización y reespecificación, es decir, se trata de una recepción mediada por una interpretación sociológica de una versión ya generalizada de teorías científicas. En el proceso de generalización se oscurecen algunas partes de la teoría original, lo que contribuye a que el problema del isomorfismo pierda fuerza. No se intenta, pues, explicar fenómenos sociales con ayuda de una teoría científico-natural, sino que ésta estimula un nuevo modo de ver los fenómenos sociales que puede desembocar en un proceso de construcción teórica independiente.

¿Pero dónde reside la atracción de los modelos científico-naturales para los sociólogos? En primer lugar, no podemos dejar de señalar que esta recepción de modelos tomados de las ciencias naturales que se ha acentuado últimamente no se debe exclusivamente a una demanda activa por parte de los sociólogos, ya que muchos científicos de renombre han tratado de aplicar sus teorías en el terreno sociológico. Es como el pescador de Goethe: a medias cogió al pez y a medias cayó él solo. De este modo, la sociología actual no está expuesta sólo a un imperialismo de las ciencias económicas nuevamente activo, sino también a las aspiraciones hegemónicas de la ciencia natural. Como veremos, la simultánea receptividad de ambas influencias no es casual. 
Por lo que atañe a la atracción de los nuevos modelos científicos para la sociología, puede ser que el carácter normativo o emocionalmente sugestivo de conceptos como autoorganización, catástrofe o caos haya elevado la receptividad, así como el deseo de emular el prestigio de las ciencias duras ${ }^{6}$. Pero más importantes son, de hecho, las semejanzas formales que saltan a la vista entre modelos científicos de dinámicas no lineales y determinados procesos sociales, pues aunque de un modo general y vago — suggestive, como dicen los americanos-, estas semejanzas alientan la esperanza de que se pueda sacar de los modelos científicos algo útil para la explicación de procesos sociales. Autores como Walter Bühl (1990), que se ha pronunciado al respecto con todo detalle, ven la relevancia sociológica de esas teorías principalmente en que ofrecen modelos para el análisis de procesos discontinuos de cambio social, ciclos, fluctuaciones y catástrofes, como reza el subtítulo de su último libro. Además, ello fomenta el estudio de procesos que han sido sistemáticamente ignorados, según Bühl, por las teorías sociológicas dominantes hasta ahora. Aunque no se comparta el punto de vista de Bühl sobre la obsesión por el equilibrio reinante en la teoría sociológica hasta nuestros días, haciendo especial referencia a Marx y a Weber, hay algo de cierto en su fundamentación del significado que tienen los modelos de dinámicas no lineales para la sociología. Mi tesis, aunque compatible con esa fundamentación, es otra, a saber: que la especial relevancia de estos recientes modelos matemáticos y científico-naturales reside en su contribución para la solución del llamado problema micro-macro ${ }^{7}$. Exactamente de este problema, del surgimiento de fenómenos macro a partir del comportamiento de los elementos del sistema sometidos a reglas reconocibles, se ocupan todas las nuevas teorías científicas que hemos mencionado. Esto es especialmente evidente en el caso del paradigma de la autoorganización. Pero también modelos matemáticos de procesos discontinuos tales como procesos de movilización o difusión generan el macroacontecimiento a partir del microcomportamiento interdependiente y descoordinado de los elementos del sistema. Lo mismo vale para modelos evolutivos del cambio en los cuales los microprocesos de la reproducción y de la mortalidad diferencial producen,

${ }^{6}$ Esto vale a pesar de que en el lenguaje coloquial al menos los conceptos de autoorganización y de catástrofe sean utilizados en un sentido distinto del matemático. Una catástrofe en el sentido de Thom no es un acontecimiento que irrumpe repentinamente dejando graves consecuencias negativas a terceros implicados y no implicados, sino un tipo determinado de transformación del perfil de singularidad en una familia de funciones bajo variación gradual de uno o más parámetros. Y la autoorganización no es un proceso de toma de decisiones sobre la base de una democracia directa para seguir metas comunes, sino un proceso natural de formación no planeada de estructuras. Para la mayoría de los sociólogos, la atracción del paradigma de la autoorganización radica justamente ahí.

7 El problema micro-macro al que nos referimos aquí no consiste en la exposición que de él han hecho recientemente Berger, Eyre y Zelditch (1989) de que hay posibles diferencias en la estructura formal que se ocupan de fenómenos micro o macro, sino que se trata de la generación de macroacontecimientos o macroestructuras mediante microprocesos, esto es, por agregación e interacción de acciones individuales. 
respectivamente, cambios estructurales y conservación de la estructura a escala poblacional.

Los propios científicos, especialmente los autores del paradigma de la autoorganización, ven que estas teorías pueden contribuir a construir un puente entre lo micro y lo macro. De una u otra forma, todos ponen de relieve que los procesos autoorganizativos generan propiedades a nivel macro de los sistemas observados que no pueden deducirse de las propiedades mensurables de sus elementos por simple agregación. En el caso del láser, por ejemplo, la nueva propiedad es un rayo luminoso de intensidad especial; en procesos hidrodinámicos como la inestabilidad de Bérnard, es un patrón determinado de movimiento; en otros casos, es la locomoción de una colonia de células o la capacidad de visión espacial. El problema micro-macro es un problema de emergencia de nuevas propiedades o un problema de interación entre niveles inferiores y superiores en sistemas jerárquicos, en los que la jerarquía no ha de ser entendida como una cadena de órdenes, sino como una relación de inclusión. Mi primera tesis es, pues, que la expectativa de una mejor comprensión de fenómenos sociales emergentes en el sentido aquí especificado es la razón más importante del atractivo no sólo del paradigma de la autoorganización, sino de otras teorías científicas de dinámicas no lineales como lo es, en último extremo, la teoría de la evolución.

Aunque no es ni mucho menos nuevo, el problema micro-macro parece gozar de una cierta actualidad en la sociología. Mi segunda tesis es que esta actualidad está relacionada con el renacimiento de enfoques centrados en la acción incluso en el ámbito de la macrosociología. Mientras el cambio estructural a nivel social global fue explicado sistémicamente como reflejo de imperativos funcionales o de modificaciones semánticas del desarrollo tecnológico o como una secuencia paulatina de impulsos en la diferenciación social, es decir, mientras las propiedades macro eran atribuidas a variables macro no era necesaria una conexión con el nivel micro de la acción individual. Tan sólo al intentar unir los dos niveles con el fin de explicar fenómenos macro (o sea, lo contrario del muy usual método de utilizar variables macro para explicar problemas micro) se plantea el problema en toda su extensión. Aunque ya Parsons hizo el intento explícito de unir en una misma teoría general el nivel micro de la acción social con el nivel macro del desarrollo de las estructuras sociales, no capta este aspecto especial de la cuestión en su concepción de la jerarquía de control y las condiciones recíprocas ni en su concepto de interpenetración. Más significativo es el concepto de "consecuencias no pretendidas de la acción» que siempre ha sido puesto de relieve como objeto central del conocimiento sociológico. Pero éstas pueden ser un problema, obviamente, si se argumenta en términos de acción o de actor, como lo pone de manifiesto una temprana y muy citada formulación del problema micro-macro realizada por Hayek: "If social phenomena showed no order except in so far as they were consciously designed, there would be ... only problems of psycology. It is only in so far as some sort of order arises as a result of individual action, but 
without being designed by any individual that a problem is raised which demands theoretical exploration» (Hayek, 1955: 39).

Sólo que Hayek era economista y los economistas se han ocupado desde hace mucho tiempo de buscar puentes de este tipo entre lo micro y lo macro. No necesito más que remitirme a Karl Menger, quien en sus investigaciones sobre el método de las ciencias sociales y de la economía política de 1883 titulaba así uno de sus capítulos: «Sobre la comprensión exacta (atomística) del origen de esos fenómenos sociales que son el resultado no premeditado de desarrollos sociales ${ }^{8}$. Por tanto, es aquí, al representarnos la emergencia de fenómenos macro a partir de acciones e interacciones en el nivel micro, donde determinados modelos económicos y modelos científicos de autoorganización encuentran un terreno común. Por su parte, al reencontrase de nuevo la sociología con la categoría de la acción social, se vuelve sensible a ambas influencias, la física o biológica y la económica. Así pues, no ha de extrañarnos que autores como Siegwart Lindenberg, afín a un paradigma explicativo de corte economicista para el comportamiento individual, hayan sido los que últimamente han producido las contribuciones más decisivas en el ámbito alemán para formular - por no decir, para resolver - el problema micro-macro (Lindenberg, 1977).

Sin embargo, la tesis de que con el renacimiento del enfoque teórico centrado en la acción, a lo que se añade la actualidad del problema micro-macro, la sociología se muestra sensible a los modelos científicos de autoorganización, tiene un fallo en su armonía, aunque tan sólo a nivel puramente descriptivo, porque no recoge los préstamos teóricos que Niklas Luhmann y algunos seguidores suyos como Gunther Teubner tomaron de la teoría de la autopoiesis de Maturana y Varela. La sociología de Luhmann no está de modo explícito orientada a la acción y, por tanto, falta en ella la variante relativa a la acción social del problema micro-macro. Tanto Luhmann como Teubner tratan fenómenos emergentes, sea la emergencia de unidades del sistema como la emergencia del mecanismo autorreproductor mismo, que es vista como una propiedad sistémica más del nivel macro9. La teoría sistémica de la autopoiesis podría plantear el problema de la emergencia social de un modo particularmente diferente $^{10}$. Sin embargo, la versión centrada en la acción del problema macromicro parte de un interés cognoscitivo precientífico, el interés por el tema del destino, del éxito o fracaso de la autoconservación colectiva del homo sapiens bajo el desarrollo progresivo de su propio potencial. Este tipo de preguntas, este miedo, es el que siempre ha estado unido al concepto de "consecuencias no pretendidas de la acción".

${ }^{8}$ Estas ideas son utilizadas de nuevo en la teoría económica de las instituciones sociales, esto es, en el neoinstitucionalismo económico. Véase Andrew Schotter (1981).

9 Véase explícitamente en Teubner (1989: 32, 42, 94).

${ }_{10}$ Teubner (1989) une los órdenes jerárquicos circularmente mediante la autorreferencialidad y del mismo modo se desunen. Aunque no ocurre de otro modo fundamentalmente cuando en el enfoque de la acción se especifica que la acción a nivel micro, que produce determinados efectos a nivel macro, a su vez se ve influida por otras propiedades sistémicas del nivel macro. 
Una vez que hemos entendido dónde reside el atractivo de los modelos físico-matemáticos para el sociólogo, se plantea entonces la pregunta siguiente: ¿hasta dónde alcanza realmente la potencia explicativa de los modelos basados en la autoorganización en el ámbito sociológico? En primer lugar, resulta evidente que los mecanismos de agregación e interacción de los sistemas físicos, químicos e incluso orgánicos no pueden ser traducidos directamente. Sin embargo, estos mecanismos son el núcleo de las teorías científicas sustantivas que sirven de modelo, por lo que su equivalente sociológico habría de tener el mismo significado en la explicación de los macrofenómenos sociales. Si se considera únicamente la estructura formal de los diversos mecanismos naturales, se perciben estimulantes posibilidades para traducir el modelo. Se puede comprobar que en fenómenos de autoorganización en sistemas físicos, químicos y orgánicos son de central importancia procesos catalíticos y autocatalíticos, así como la acción conjunta o la interferencia de reacciones recíprocas entre elementos que circulan con distinta velocidad y que ejercen sobre el sistema una acción condensadora o ratificante. Pero estos paralelismos no nos llevan muy lejos mientras no podamos decir, refiriéndonos a nuestro objeto, qué es lo que se condensa o dispersa a tal intensidad y tal frecuencia y mediante cuáles interacciones entre personas humanas. Es decir, estos paralelismos, en principio, nos señalan torpemente — aunque ya es algo- la dirección en la que podríamos buscar.

Con todo, no hay una frontera determinable a priori para la traducción de modelos científicos efectuada mediante abstracción y reespecificación. Se encuentra allí donde las premisas centrales de las teorías físicas y químicas dejan de tener validez incluso en un sentido figurado. Éste es el caso de la premisa de la invarianza espacial y temporal de los elementos ${ }^{11}$. Mientras que los sociólogos, por regla general, son conscientes de esta premisa y de la problemática que encierra, hay otra específica de los modelos autoorganizativos que rara vez se hace explícita: el hecho de que se trata de sistemas de gran número de partículas. Los modelos matemáticos correspondientes se basan precisamente en esto.

En la realidad social hay una variedad de situaciones en las que se cumplen las dos premisas mencionadas para el surgimiento de discontinuidades y procesos de autoorganización en el sentido de los modelos científicos que hemos tratado aquí. En ella encontramos procesos, con un alcance espaciotemporal naturalmente limitado, centrados en la conducta colectiva de grandes cuasigrupos cuyos elementos -individuos, presupuestos o incluso organizaciones - pueden estar cultural o normativamente regulados respecto del aspecto de la conducta que nos interesa, actúan en constante interferencia mutua, pero no están organizados, es decir, su conducta no está coordinada

11 «Las ciencias naturales se basan en la constante intercambiabilidad de los fenómenos investigados, es decir, en que no son individuos singulares ni temporalmente únicos» (Markl, 1989: 133). 
con arreglo a un plan de acción. Pueden encontrarse ejemplos no sólo en el ámbito del típico comportamiento de masas, sino también en procesos de formación de la opinión pública, de la movilización política, en los ciclos de coyuntura de temas políticos o de la moda. A esta categoría pertenecen también los procesos de estructuración espontánea del espacio y los cambios cíclicos en la elección de profesión, así como en general todos los procesos que pueden describirse como procesos de mercado o que tienen que ver con situaciones de competencia en grandes poblaciones. Puede que siempre haya una simplificación, pero de no ser así sería imposible describir en su totalidad el comportamiento de las unidades recurriendo a reglas relativamente sencillas que permanezcan estables durante todo el período al que la investigación se refiere.

El ámbito de aplicación de los modelos de generación de estructuras y rupturas de estructuras no carece de importancia, e incluso podrá crecer aún debido a la creciente desaparición del vínculo tradicional y a la erosión de las relaciones jerárquicas en las sociedades modernas ${ }^{12}$. Crecería en importancia a medida que surgiesen procesos análogos al funcionamiento del mercado y podría justificar en parte el atractivo creciente que en estos momentos tienen los modelos económicos para la sociología. Al concentrarse explícitamente en las consecuencias del comportamiento colectivo, y con ello en la especificación de los mecanismos de interacción sobre los que se basan los macroefectos, los modelos autoorganizativos (y los modelos de procesos no lineales de ruptura del orden) pueden llegar a ser un contrapunto de la tendencia de los sociólogos de la acción social por concentrarse en la explicación de decisiones individuales ${ }^{13}$.

Sin embargo, me atrevo a sostener — y ésta es mi tercera y última tesisque con el uso de modelos teóricos de comportamiento colectivo para explicar macrofenómenos sociales no se capta la problemática central de la dinámica social. La razón decisiva de ello no es tanto el hecho de que la premisa de la invarianza en el ámbito social deja de cumplirse allá donde se den procesos de aprendizaje en virtud de los cuales las reglas vigentes de comportamiento se sustituyen por otras y, además, el modo en que esta sustitución opera no puede, a su vez, ser cifrado en una nueva regla sencilla de comportamiento colectivo, por lo que no resulta predecible ni modelizable. Cierto, los seres humanos aprenden de las consecuencias de su comportamiento colectivo y la próxima vez reaccionan ante la misma situación de modo distinto, de suerte

${ }^{12}$ Véase al respecto el argumento de Esser (1989) basado en la tesis de Beck de la individualización. Aunque para que el ámbito de aplicación de los modelos de conducta colectiva pueda crecer realmente no puede perderse con la individualización esa invarianza del comportamiento elemental que se presupone en los modelos de autoorganización. Individualización no significa necesariamente idiosincrasización. Ya M. Weber observó que no sólo normas y tradiciones, sino también la combinación de intereses y oportunidades, hacen predecible y estructuran la acción social.

13 Para Esser (1989), también el núcleo nomológico de una sociología que quiera dar cuenta del fenómeno social de la individualización es una teoría de la acción que pueda explicar actos electivos individuales a partir de reglas de conducta reconocibles. 
que muchos efectos agregados previstos no aparecen. Incluso la mera anticipación de determinadas consecuencias puede bastar para que el comportamiento colectivo tome otras direcciones. Lo difícil que nos resulta predecir tales anticipaciones de cuasigrupos relevantes y sus efectos sobre la modificación del comportamiento colectivo hasta ese momento conocido lo hemos podido comprobar, una vez más, en el contexto de los acontecimientos ocurridos en la antigua RDA. Nuestra situación es más difícil que la de la ciencia económica, a la que F. A. Hayek, bajo el bonito título de "Pretence of Knowledge», le reprochó su actitud de ingenuo cientifismo al equiparar lo mensurable con lo importante y, de este modo, llegar a afirmaciones manifiestamente falsas sobre, por ejemplo, el desarrollo del desempleo y la coyuntura ${ }^{14}$.

Más aún que la especial capacidad humana de aprendizaje, hay otra razón que limita la relevancia de modelos emergentistas procedentes de las ciencias naturales para la sociología. Al contrario que para los sistemas que estudian las ciencias naturales, en las ciencias sociales no sólo rige la «mano invisible» de Adam Smith (o la astucia de la razón de Hegel), sino también el schilleriano «en tu pecho están las estrellas de tu destino». Los seres humanos son capaces de organizarse y de fijarse metas colectivas. La existencia de poderosos actores corporativos es una consecuencia de ello. Intervienen o, al menos, lo intentan cuando el resultado anticipado de procesos de configuración estructural, de fluctuaciones, círculos viciosos y espirales no les parece deseable. De este modo, los procesos espontáneos, naturales, de conducta colectiva son desviados permanentemente. Por ello, sólo muy pocos macroacontecimientos o macroestructuras son realmente fenómenos emergentistas puros en el sentido del paradigma científico. Esto puede mostrarse bien tomando el sistema de la ciencia como ejemplo. Krohn y Küppers (1989) han analizado la producción y la reproducción de ese sistema en cuanto sistema autoorganizado que resulta de la acción investigadora en equipo. Este planteamiento es sostenible mientras que se hable de la ciencia en cuanto sistema cognitivo, pero no vale para explicar la estructura institucional de un sistema científico concreto y sus transformaciones. Sobre todo, los macroefectos potencialmente destructivos y no pretendidos no son preferentemente el resultado de procesos que siguen el paradigma científico del surgimiento espontáneo del orden o de la ruptura espontánea de éste ni pueden ser concebidos a partir de ellos. Pueden ser igualmente el resultado de una mala dirección o de interacciones del tipo del «dilema del prisionero" y otras situaciones de interdependencia estratégica con carácter de trampa ${ }^{15}$. Para explicar este tipo de interacciones no sirven de

14 Véase Hayek (1989). «In the explanation of the working of such structures we can ... not replace the information about the individual elements by statistical information, but require information about each element if from our theory we are to derive specific predictions..." (1989: $4)$. « ... this failure of the economist ... is closely connected with their propensity to imitate as closely as possible the procedures of the brilliantly successful physical sciences...» (1989: 3).

15 Véase, por ejemplo, la "trampa de los entrecruzamientos políticos» que ha analizado Scharp (1988). 
ayuda los modelos autoorganizativos porque ya no se trata de sistemas de partículas.

Acción planificada, interacción estratégica y procesos de comportamiento colectivo - las tres formas de orden con las que opera la macrosociologíanecesitan no sólo planteamientos teóricos respectivamente distintos. El reto teórico decisivo radica, a mi parecer, en el análisis de la interferencia entre procesos de comportamiento colectivo, por un lado, y las interacciones estratégicas de los actores corporativos junto a las reacciones dirigidas en su contra, por el otro. El problema de la interferencia así esbozado diferencia - y complicala dinámica de búsqueda de soluciones y de generación de problemas derivados que desarrollaba la teoría de sistemas del primer Luhmann. Justo porque lo que se requiere es la combinación e integración de distintos planteamientos teóricos cuando la mayoría de los investigadores tienden a moverse en el marco de un paradigma científico, la sociología no ha podido ofrecer mucho hasta ahora para solucionar el problema de la interferencia; más aún, incluso la identificación misma de este problema puede uno buscarla en balde en las manifestaciones programáticas de los sociólogos. Por otro lado, ocasionalmente, aparecen trabajos empíricos en los que se investiga la interferencia que se da en la práctica entre procesos de comportamiento colectivo, acción política y acción de las organizaciones. Al respecto es ejemplar el trabajo de Wolfgang Streeck sobre los problemas organizativos de los sindicatos en el Estado social democrático (1981); trabajo que el propio autor, de modo significativo, no lo sitúa explícitamente en el marco de las reflexiones metodológicas fundamentales del tipo mencionado.

Para Streeck, los sindicatos, como todas las organizaciones, son creaciones espontáneas, no planificadas, orgánicas, pero al mismo tiempo son también un conjunto de funciones desarrolladas para un fin (Streeck, 1981: 13). Son, pues, efecto e instrumento de los procesos de movilización. La evolución de los sindicatos, de acuerdo con esto, es el producto de la interacción causal entre las probabilidades y las estrategias de movilización, esto es, entre la disposición de los potenciales miembros del sindicato para la acción colectiva y la acción estratégica de la organización. Las probabilidades de movilización se reducen, según Streeck, como consecuencia de los cambios en la estructura social, pero también como reacción a la transformación del significado de los sindicatos producida por iniciativa legal ${ }^{16}$. A esta transformación exógena de sus probabilidades de movilización, los sindicatos reaccionan bien intentando fortalecer los motivos para sindicarse de sus potenciales miembros en vista de su situación social, o bien elevando su atractivo para miembros potenciales. Así queda el círculo cerrado: acción política, acción de la organización y la disposición

${ }^{16}$ Así, el atractivo de los sindicatos baja si como consecuencia de reglamentos de tarifas a los no miembros no se les puede dejar fuera de los frutos de una exitosa lucha por los intereses que representan. Al mismo tiempo, el desmantelamiento por parte del Estado del sistema de seguridad social ha disminuido constantemente el significado de la subvención a los sindicatos (Streeck, 1981: 67). 
para la acción colectiva determinada por la situación social del cuasigrupo de referencia se entrelazan al máximo, influyéndose mutuamente, en un período de tiempo. Tales interreferencias eran, y todavía lo son, fácilmente observables en la radical y rápida reestructuración de la antigua RDA. El interés de la teoría sociológica radicaría en buscar algunos modelos interreferenciales capaces de generalización. Pero, llegados a este punto, los modelos científicos que hemos tratado no pueden ayudarnos más, pues ahí llegamos a un límite definitivo para el aprendizaje mediante préstamos teóricos de una ciencia a otra.

\section{BIBLIOGRAFÍA}

Berger, Josef; Eyre, Dana P., y Zelditch, Maurice, Jr. (1989): "Theoretical Structures and the Micro-Macro Problem», en Josef Berger, Maurice Zelditch Jr. y Bo Andersen (eds.), Sociological Theories in Progress, Newbury Park, pp. 11-32.

BüHL, Walter L. (1990): Sozialer Wandel im Ungleichgewicht. Zyklen, Fluktuationen, Katastrophen, Stuttgart: Enke.

EsSER, Hartmut (1989): «Verfällt dir "soziologische methode”?», en Soziale Welt, 40: 57-75.

HAKEN, Hermann (1978): Synergetics. An introduction, Berlín: Springer.

HaYeK, Friedrich A. (1955): The Counter-Revolution of Science, New York: Free Press.

- (1989): "The Pretence of Knowledge», en The American Economic Review, 79, 6: 3-7.

KroHn, Wolfgang, y KüPpers, Günter (1989): Die Selbstorganisation der Wissenschaft, Frankfurt: Suhrkamp.

LindenberG, Siegwart (1977): «Individuelle Effekte, kollektive Phänomene und das Problem der Transformation", en Kurt Eichner y Werner Habermehl (eds.), Probleme der Erklärung sozialen Verhaltens, Meisenheim am Glan: Hain, pp. 46-84.

LuHMann, Niklas (1984): Soziale Systeme. Grundriss einer allgemeinen Theorie, Frankfurt a.M.: Suhrkamp. (Hay traducción castellana en FCE.)

MARKL, Hubert (1989): «Sind die Sozialwissenschaften Naturwissenschaft?», en Wisseschaft zur Rede gestellt. Über die Verantwortung der Forschung, München/Zürich: Piper, pp. 119-144.

MAYNTZ, Renate (1990): The Influence of Natural Science Theories on Contemporary Social Science, MPIFG Discussion Paper 90/7, Köln: Max-Planck-Institut für Gesellschaftsforschung.

QueISSNER, Hans (1987): Kristalline Krisen: Mikroelektronik - Wege der Forschung, Kampf um Märkte, München: Piper.

SCHARPF, Fritz W. (1985): «Die Politikverflechtungsfalle: Europäische Integration und duetscher Föderalismus im Vergleich", en Politische Vierteljahresschrift, 26: 323-356.

SCOTTER, Andrew (1981): The Economic Theory of Social Institutions, Cambridge: Cambridge University Press.

STREECK, Wolfgang (1981): Gewerkschaftliche Organisationsprobleme in der sozialstaatlichen Demokratie, Königstein/Ts.: Athenäum.

Thom, René (1972): Stabilité structurelle et morphogénèse, Reading, Mass.: Benjamin. (Hay traducción castellana en Gedisa.)

Teubner, Gunther (1989): Recht als autopoietisches System, Frankfurt/Main: Suhrkamp.

(Traducción de Javier RODRÍGUEZ MARTíNEZ.) 


\section{ABSTRACT}

The methodological relations between natural and social sciences are the object of a renewed debate within the bosom of the latter. Over the last two decades, evolution in natural science has produced sufficient signs for us to be able to talk about the emergence of a new paradigm that would differ from the Newtonian physical science model. From the vantage point of philosopy, sociological theory and methodology has advocated that such advances in natural science would have their repercussion on social science. Renate Mayntz carried out research work in the Max Planck Institute, the principal results of which can be seen in this article. His pessimistic thinking in respect of the possibilities of a methodological transfer from one sphere to another does not prevent him from giving a proper evaluation of the sociological research areas where an effective methodological influence has occurred. 